\title{
Care Robots: From Tools of Care to Training Opportunities. Moral Considerations
}

\author{
Maurizio Balistreri ${ }^{1(\bowtie)}$ and Francesco Casile ${ }^{2}$ \\ ${ }^{1}$ Department of Philosophy and Educational Sciences, University of Turin, \\ Turin, Italy \\ maurizio.balistreri@unito.it \\ ${ }^{2}$ Bioethics Committee, University of Turin, Turin, Italy
}

\begin{abstract}
New technology should not perceived as a threat: on the contrary, it is a resource. It is our job to use it in the most appropriate way. Moreover, new technology can make a significant contribution to nurse training: for example, immersion into virtual reality with a visor and a simple application does not only allow one to experience fantastic adventures, but also to enjoy a relationship with the patient through simulation. Also, virtual reality can promote patient/teacher interaction: both, for example, can be projected or immersed in virtual reality, or the teacher can project his 'virtual' image into a real scenario. However, robots too could contribute to training nursing staff: health operator training courses today widely use dummies which are appropriately planned for standing training. They are increasingly true-to-life, favouring empathy with the clinical situation simulated each time and allowing the student to exercise not only technical abilities, but also critical thinking, the ability to work in a team and communication skills. We shall examine some moral questions linked to the increasingly frequent use of human-faceted robots to train nursing staff.
\end{abstract}

Keywords: Carebots $\cdot$ Robots $\cdot$ Bioethics

\section{Robots in Caring and Healing Roles}

Over the last few decades, the use of robots has markedly increased and it is easy to predict that they will be even more a part of our existence in the decades to come. Robots also promise to be a significant resource in the field of medicine: especially patients may derive advantages from their introduction and use, in that they may be assisted by machines that are not subject to tiredness or stress, hence may be operative $24 / 7$ without a break. Further, robots may not only monitor their health condition more precisely than a health operator or human (for example, robots could be programmed to note a significant change in the behaviour, habit or voice of the person followed), but also compensate for or limit the impact of some physical disabilities. They could, furthermore, assist a sick or elderly person just as well as a human: for example, there are robots that can be programmed to remind the patient when it is time to take medicine and which pharmaceuticals out of many to take, whilst others have the task of stimulating their cognitive abilities through games or questions. In this way, they can function as a both physical and cognitive prosthesis, in that a person who, for example, 
forgets to eat will be on the road to ruin in many ways, not least cognitively. And this type of thing may become a vicious circle [1]: the person continues not to eat, lucidity worsens, he forgets to eat again or take care of himself [2].

However; the use of robots in the field of care may also have important repercussions for health operators, as well as for anyone beside a fragile person, in that caregivers could have at their disposal a very useful tool [1] to use for activities that are more repetitive (for example, bringing medicines to the patient's bed, handing out breakfasts, lunches and dinner) and tiring (for example, lifting a patient from bed or helping him walk and exercise or work) [3]. In these terms, carebots could help people carry out their care job without getting ill or still having to take on unbearable burdens that are incompatible with a satisfying life with their family or those held dear: "caregiving practice" - writes Shannon Vallor - "in the absence of adequate resources and support have a highly destructive impact on emotional, prudent and social capacities - exasperated stress, anxiety, physical and exhaustion can cause emotional withdrawal, a gradual numbing of affection or extreme emotional volatility - one or all of these consequences can cause fractured relationships with those assisted and others, serious depression, motivational apathy, compromise ability of judgement and even lead to a total nervous breakdown [3]." It is true that the most well-to-do have always been able to choose to delegate part of their care work to their family or those they are responsible for (not only the elderly, but also children and individuals displaying disabilities) to others (not only care institution staff, but also caregivers or freelance workers) who see their work force and skills in care for others. However, the spread of robots could benefit the whole population, in that their cost could notably lower over time. Further, any initial expense for their purchase could be amortised over a period, in that they do not have to pay the robot a wage or overtime. And once purchased, the robot can be resold or possibly shared with others who need care and assistance. For this reason, robot technology could contribute to promoting social justice [1]", in that the care work would no longer be an activity imposed by circumstance, but the result of a freer choice that could be shared with those most interested.

Of course, spreading robots in the field of care also arouses worry. For example, there is the fear that with the use of increasingly intelligent robots, patients could be totally abandoned to assistance from machines; and at that point, human beings would - maybe forever - lose empathy, in that they would no longer get to experience others' suffering. However, we cannot imagine that robots, once introduced into the field of care, would substitute the health staff thus far employed fully and in all services. Robots may reveal themselves as able to replace health operators in those contexts requiring more simple and repetitive services (for example, supplying meals or medicine to patients at times that could be programmed), but not in those care activities needing to respond to more complex situations (not only diagnosis of illness and choice of type of operation or therapeutic treatment, but also the psychological assistance of the patient or elderly person). So the robots could provide a "superficial" or mechanical service [4] - for example performing repetitive manual tasks -, while the health operators (doctors and nurses) "deeper" care for the patient's specific individuality and needs: "While we have no reason to think that robots will be unable to offer individuals superficial, we share Coeckelbergh's scepticism regarding the possibility that they may go beyond superficial care and offer that type of reciprocal company or friendship that 
characterises deep care. (...) Robots' inability to offer deep care does not hinder human caregivers from using them to offer good care, respecting dignity [1]”. Further, introducing carebots into medicine could open up to assistance and care new possibilities that we still struggle to imagine and which produce not the disappearance but the redefinition of care practices (least of all those we perform to meet the needs of others) [4]. For example, can we think that thanks to the very help of robots, elderly or ill people could count on better quality relationships with their family? People responsible for the care of a sick or elderly person (it does not matter whether they are family or paid by the family or an institution) can sometimes suffer from depression and discouragement or be overwhelmed by work and so not have the time or energy to dedicate to entertainment (to give the person they care for a pleasant, fun time). With robots programmed to carry out the more tiring, boring care work, things could change, in that family members or in any case others could use part of their time in relating to people who are not self-sufficient. It is also possible that part of the care activity would consist of laboratory planning of machines to deal with care and assistance. Indeed, one can imagine that robots should be personalised to take into account the needs and preferences of those they will then care for (after all, different patients will not only have different needs in terms of therapy, but also different habits, which the robot will have to take into account). It will therefore be the family members' task - as well as the health operators', of course - to rebuild the profile of the person and indicate the tasks and services the robot will have to perform. Of course, people who are still competent may choose with greater freedom the type of robot they want and the type of activity they can expect. However, the interests of those who are incompetent - unless perhaps they have left a trail of their own volition - must be safeguarded by others.

\section{Interactive Robots for Training and Refining Clinical Skills}

New technology should not be perceived as a threat: on the contrary, it is an important resource. It is up to us to use it in the most appropriate way. Carebots are more and more present by the patient's bed and can collaborate in care work. However, new technology can make a significant contribution to nurse training: for example, immersion into virtual reality with a visor and a simple application does not only allow one to experience fantastic adventures, but also to enjoy a relationship with the patient through simulation. Also, virtual reality [5, 6] can promote patient/teacher interaction: both, for example, can be projected or immersed in virtual reality, or the teacher can project his 'virtual' imagine into a real scenario and assist health operators. However, robots too could contribute to training nurses and give them the chance to practice clinical skills before they see a real patient. Health operator training courses today widely use dummies which are appropriately planned for standard training [7-9]. The models' physical and anatomical features are increasingly true-to-life, favouring empathy with the clinical situation simulated each time and allowing the student to exercise not only technical abilities, but also critical thinking, the ability to work in a team, and appropriate communication skills. One can imagine that increasingly intelligent and interactive robots could make training even more useful for refining clinical skills. Robots to train have already been constructed so as to let doctors and training 
nurses carry out plenty of tests, like measuring blood pressure or checking pulses and breathing [10]. Further, it can be much easier for teachers to check the student's level, in that the automaton is connected to a computer or able to register its results in various simulation tests: a "robot can be programmed to simulate the required actions in a way that can be objectively and quantitatively measured [7]." Also, according to didactic needs, the robots could be programmed to interpret people of different ages and different character profile: for example, the robot Hal, created by Gaumard, not only looks like a child, but also behaves like one: tracking a finger by eye is not his sole ability; he can also reply to questions, cry for his mother and feel anaphylactic shock. In this way, the student could face up to different situations and practice in working in pediatric conditions where the patient's compliance is partially or completely absent.

However, robots are not only useful in practising the student's technical skills: especially in basic training paths, robots could also be used to improve health operators' readiness to understand the patient's feelings more precisely (empathy). We are now used to 'mechanisms' which present themselves with sentiments and needs: which do not want to play with riddles, but say if they are hungry or sad and must be nourished, entertained and cleaned because otherwise they would die or not grow. It does not seem difficult to project a robot able to simulate the behaviour of a patient and with different reactions according to the way in which it is treated: for example, if the operator's care is adequate, it could smile or say thank you; but if it is neglected, it could cry or get angry and sad and even say, "You are mistreating me." To programme it, it would just be necessary to gather information relating to the quality or enjoyment of care, obtained by the patients via questionnaires or interviews: in this way, we will get to know their expectations regarding the health operators, what is important in them for care and what is, on the other hand, neglected. Of course we already have an abundant literature regarding the most appropriate behaviour towards a patient: but perhaps, if we want to build a robot able to simulate the behaviour of a patient, it will be necessary to develop thus far unconsidered aspects. Through interaction with robots, the health operator would first and foremost have a chance to evaluate immediately the appropriateness of its care, with no risk of self-deception or not adequately grasping the patient's reactions. How many patients are brave enough to complain about a lack of care? Moreover, children may not be forthcoming about their symptoms: they can often do so by facial expression or behaviour and gestures. While students carry out treatment, the robot could react to the prick of a needle by crying or expressing displeasure. He might also move, not only showing emotions and sentiments, but mimicking the difficulty often found when treating a person in pain. Further, due to illness, some people are unable to react to what is going on around them, and seem unable to have feelings: on the contrary, the robots will not feel any awe towards us or have any embarrassment and shame, in that - in any situation - they will do what they were programmed to display. Overall, we can self-deceive with people, but with robots this is more difficult. For this reason, health operators inter-reacting with robots can learn to calibrate their behaviour or relationship with the patient, and then modify it in the face of negative responses: once the robot starts to seem content and is no longer agitated, crying or complaining, then here we are: we have reached the adequate care level.

We can add to this that training with robots could make it easier for health operators to accept robots for care and assistance, and to recognise that robots are a resource that 
can not only lighten health operators' work but also improve the quality of care. We are actually at the start of a revolution in the field of training that could radically alter training nursing staff and doctors. Of course, we have not yet fully assessed the effectiveness of using robotics in training, but we do have cause for optimism [11-14]. Studies demonstrate that undergraduate student nurses can substitute as much as half of their clinical hours by simulations without affecting their ability to pass their nursing exam and complete their study path with flying colours. In fact, new research indicates that up to $50 \%$ of clinical hours can be replaced with simulation training $[15,16]$.

\section{Robots for Training and Moral Issues}

Over the last decades, many people have grown affectionate and constantly taken care of electronic devices as elementary as, for example, Tamagotchi: one can imagine that the more robots resemble human beings, the more we will worry for their wellbeing and it will be far easier to 'empathise' with them. Despite being 'corporeal' enough for a child to imagine its death, Tamagotchi is still a virtual creature in a plastic egg, whilst robots are machines that are increasingly taken for human beings. In these terms, interaction with these robots raises the same questions that emerge in relation to the use of robots in the field of care and affectionate relationships.

We do not yet know if our character can be affected by interaction with a robot: for example, there is the worry that, over time, violent behaviour toward a machine may turn one towards violence. Think, for example, of sex robots: could the use of sex robots programmed to refuse sexual relationships increase violence against women? And could someone spending a lot of time before a computer killing, raping and torturing other 'people' be then more likely to commit such crimes in real life? This is a complex question without scientific agreement: the possibility that care towards robots could promote empathy is only a marginal research theme, but it deserves greater attention, because the risk is that one starts to feel affection and tenderness towards a robot. For some people, the experience with the robot could turn out to be a disappointment, whilst for others interaction with the robot could seem an authentic relationship. For example, this is why Gaumard did not want to make its robot Hal too realistic and like a flesh-and-blood child. They feared such a robot that could also bleed to death or experience cardiac arrest might be too emotionally traumatic for student just being introduced to emergency pediatric situations". That is, in the field of training, robots are useful to let students implement their knowledge and learn from their mistakes. However, with overly realistic model students could get into difficulty, in that they could be afraid of erring and inflicting 'suffering' on the robot. Further, an excessively realistic android could distract participants during a tense scenario: their attention could be taken by irrelevant model details or they could carry out certain actions only to see the reaction caused. Moreover, if the operator interacting with the robot spends a lot of time with the machine or gets close to it, because he intends to care for it [17], this could negatively affect family relationships: a partner may be jealous; a child or friend could feel neglected. Virtual entities like Tamagotchi or LovePlus "girls" are not "real", but those who interact with them daily succumb to the same appeal and infatuation that children usually feel towards their favourite toys. 
Moreover, the same phenomenon has been observed studying the use of robots in the field of caring for and assisting the elderly. These people are aware that the robot is not a human being, but for them the robot is unlike a mere machine and they think they have responsibility towards it. Also, people sometimes grow so fond of a doll that they end up loving it: a lot of imagination is not required to imagine what could happen tomorrow with robots that are increasingly intelligent, technological and maybe indistinguishable from ourselves. If the robot can produce the same levels of experience and company as a human, we may not resist the temptation to treat them like authentic human beings.

But the android could also arouse completely different reactions in the human being: it is the Uncanny Valley problem [18-21]. Indeed, it has been observed that the more human the robot seem and the more similarly they behave - but not in exactly the same way - to human beings, the easier it is to feel a great sense of revulsion and disgust towards them. A possible explanation for the phenomenon, first documented by Masahiro Mori [22], is that it would mean an adaptive reaction selected by evolution as useful to keeping our distance from ill or strange people (after all, the same repulsion is felt towards cadavers and zombies), but another hypothesis has been put forward too: the humanoid robot's unnatural movements would arouse thoughts of death in us [23]. Of course, this problem could make the use of robots as training tools for nurses in basic courses or even later more difficult. The simplest solution would seem to be making the robots less similar to human beings, but in this way students would probably have less chance to test and exercise their technical abilities. However, not only has Mori's theory been inadequately studied so far, but the repulsion and disturbance observed in the Uncanny Valley phenomenon could disappear with robots completely undistinguishable from human beings. Indeed, "a 'person in health' (or 'someone' perceived as a person in health) arouses a feeling of full acceptance, empathy and familiarity in the perception of another human being. A robot undistinguishable from a human being or person in perfect health does not yet exist, but will exist in the relatively near future [24]."

\section{Conclusions}

With the development of artificial intelligence, some care and assistance activities currently carried out by nursing staff will probably be performed by intelligent robots: but in care, humans can never be fully replaced by machines. Not only can humans appear irreplaceable for some activities (I am thinking, for example, of those situations where we feel the need to have at our side one who understands us and can empathise with our feelings), but it would still be up to us to programme even the most intelligent robots. In the light of these sci-fi scenarios that are now profiled on the horizon, it also becomes important for health operators to receive the most appropriate training to be ready to face the challenges the future holds. The question is not to fix whether new technology is morally acceptable, but for which tasks we can use intelligent systems without creating a risk for our humanity and at the same time promoting the good of the patient. Further, robots could be a significant resource in both everyday practice and training. Here is no incompatibility of principle between robot technology and care 
activity: on the contrary, with the help of robots we could cultivate that disposition to care and that empathy that will probably always remain our prerogative.

\section{References}

1. Borenstein, J., Pearson, Y.: Robot caregivers: harbingers of expanded freedom for all? Ethics Inf. Technol. 12, 277-288 (2010)

2. Robin, B., et al.: Robotic assistants in therapy and education of children with autism: can a small humanoid robot help encourage social interaction skills? Univ. Access Inf. Soc. 4, 105-120 (2005)

3. Vallor, S.: Carebots and caregivers: sustaining the ethical ideal of care in the 21 st century. Philos. Technol. 24(3), 251-268 (2011)

4. Coeckelbergh, M.: Health care, capabilities, and ai assistive technologies. Ethical Theory Moral Pract. 13, 181-190 (2010)

5. Bouchard, S., et al.: Effectiveness of virtual reality exposure in the treatment of arachnophobia using 3D games. Technol. Health Care 14(1), 19-27 (2006)

6. Garcia-Palacios, A., et al.: virtual reality in the treatment of spider phobia: a controlled study. Behav. Res. Ther. 40, 983-993 (2002)

7. Huang, Z., et al.: Impact of using a robot patient for nursing skill training in patient transfer. IEEE Trans. Learn. Technol. 10(2), 355-366 (2017)

8. Maidhof, W., Mazzola, N., Lacroix, N.: Student perceptions of using a human patient simulator for basic cardiac assessment. Currents Pharm. Teach. Learn. 4(1), 29-33 (2012)

9. Moodley, T., Gopalan, D.: Airway skills training using a human patient simulator. Southern African J. Anaesth. Analg. 20(3), 147-151 (2014)

10. Yinka, B.: Pediatric robot patient offers new level of realism for doctors in training. Medical Press, 10 September 2018. https://medicalxpress.com/news/2018-09-pediatric-robot-patientrealism-doctors.html

11. Tan, G.M., Ti, L.K., Suresh, S., Ho, B.S., Lee, T.L.: Teaching first-year medical students physiology: does the human patient simulator allow for more effective teaching? Singapore Med. J. 43(5), 238-242 (2002)

12. Alinier, G., Hunt, B., Gordon, R., Harwood, C.: Effectiveness of intermediate-fidelity simulation training technology in undergraduate nursing education. J. Adv. Nurs. 54(3), 359-369 (2006)

13. Crofts, J.F., Bartlett, C., Ellis, D., Hunt, L.P., Fox, R., Draycott, T.J.: Training for shoulder dystocia: a trial of simulation using low-fidelity and high-fidelity mannequins. Obstet. Gynecol. 108(6), 1477-1485 (2006)

14. Cioffi, J., Purcal, N., Arundell, F.: A pilot study to investigate the effect of a simulation strategy on the clinical decision making of midwifery students. J. Nurs. Edu. 44(3), 131-134 (2005)

15. La Cerra, C., et al.: Effects of high-fidelity simulation based on life-threatening clinical condition Scenarios on learning outcomes of undergraduate and postgraduate nursing students: a systematic review and meta-analysis. BMJ Open 9(2) (2019). https://doi.org/10. 1136/bmjopen-2018-025306

16. Persico, L.: A review: using simulation-based education to substitute traditional clinical rotations. JOJ Nurs. Health Care 9(3), 1-7 (2018). https://doi.org/10.19080/JOJNHC.2018. 09.555762 
17. Sharkey, N., Sharkey, A.: The rights and wrongs of robot care. In: Lin, P., Abney, K., Bekey, G. (eds.) Robot Ethics: The Ethical ans Social Implications of Robotics, pp. 267282. MIT Press, Cambridge (2011)

18. Wang, S., et al.: The uncanny valley: existence and explanations. Rev. Gen. Psychol. 19(4), 393-407 (2015)

19. MacDorman, K.F., Ishiguro, H.: The uncanny advantage of using androids in cognitive and social Science research. Interact. Stud.: Soc. Behav. Commun. Biol. Artif. Syst. 7, 297-337 (2006)

20. Saygin, A.P., Chaminade, T., Ishiguro, H., Driver, J., Frith, C.: The thing that should not be: predictive coding and the uncanny valley in perceiving human and humanoid robot actions. Soc. Cogn. Affect. Neurosci. 7, 413-422 (2012)

21. Kanda, T., Hirano, T., Eaton, D., Ishiguro, H.: Interactive robots as social partners and peer tutors for children: a field trial. Hum.-Comput. Interact. 19(1-2), 61-84 (2004)

22. Mori, M.: The uncanny valley (1970). Energy 7(4), 33-35 (2012)

23. Mc Dorman, K.: Androids as an experimental apparatus: why is there an uncanny valley and can we exploit it? In: CogSci2005 Workshop: Toward Social Mechanisms of Android Science, pp. 108-118. Stresa (2005)

24. Carpenter, J.: Deus Sex Machina: loving robot sex workers and the allure of an insincere kiss. In: Danaher, J., McArthur, N. (eds.) Robot Sex. Social and Ethical Implications, pp. 261-287. The MIT Press, Cambridge (2017) 